\title{
FOURIER COEFFICIENTS OF EISENSTEIN SERIES OF ONE COMPLEX VARIABLE FOR THE SPECIAL LINEAR GROUP
}

\author{
BY
}

\section{A. TERRAS}

\begin{abstract}
The Eisenstein series in question are generalizations of Epstein's zeta function, whose Fourier expansions generalize the formula of Selberg and Chowla (for the binary quadratic form case of Epstein's zeta function). The expansions are also analogous to Siegel's calculation of the Fourier coefficients of Eisenstein series for the symplectic group. . The only ingredients not appearing in Siegel's formula are the Bessel functions of matrix argument studied by Herz. These functions generalize the modified Bessel function of the second kind appearing in the Selberg-Chowla formula.
\end{abstract}

1. Introduction. We consider some Eisenstein series for the group $S L(n, \mathbf{R})$ of $n \times n$ real matrices of determinant 1 , the special linear group. The simplest such function is the Epstein zeta function of an $n$-ary positive definite (real) quadratic form $S$, defined by

$$
\tilde{Z}(S, \rho)=\frac{1}{2} \sum_{a \in Z^{n_{-0}}} S[a]^{-\rho}=\frac{1}{2} \zeta(2 \rho) \sum_{a \in Z^{n}-0 ; \mathrm{g} \cdot \mathrm{c} . \mathrm{d} .(a)=1} S[a]^{-\rho},
$$

where the first sum is over column vectors $a$ with integer entries and the second sum is over column vectors $a$ such that the entries are relatively prime integers. Here $\zeta(x)$ is the Riemann zeta function and $\rho$ is a complex number with $\operatorname{Re} \rho>$ $1 / 2 n$ for convergence of the series. A useful formula for this function is that of Selberg and Chowla, (1.3) below, which has generalizations in [16]. This can be used to prove Kronecker's limit formula and has applications in number theory (e.g., [2], [4], and [14]). Considering the Epstein zeta function as an Eisenstein series on $\operatorname{SL}(n, \mathbf{R})$ as is noted in [3], the formula of Selberg and Chowla is nothing more than the computation of the Fourier expansion in the case $n=2$ with respect to the subgroup of $S L(2, \mathbf{R})$ of matrices of the form $\left(\begin{array}{ll}1 & x \\ 0 & 1\end{array}\right)$. For multiplying such matrices corresponds to adding the $x$ 's and $\widetilde{Z}(S, \rho)=\widetilde{Z}\left(S^{\prime}, \rho\right)$, where

$$
S^{\prime}=\left(\begin{array}{ll}
1 & 0 \\
x & 1
\end{array}\right) S\left(\begin{array}{ll}
1 & x \\
0 & 1
\end{array}\right),
$$

Presented to the Society, January 16, 1974; received by the editors October 15, 1973. AMS(MOS) subject classifications (1970). Primary $10 \mathrm{D} 99$.

Key words and phrases. Fourier coefficients, Eisenstein series, Epstein zeta function, special linear group, positive definite symmetric matrices, Bessel functions of matrix argument. 
for any integer $x$. Now use the diagonalization $[13$, p. 24]

$$
S=\left(\begin{array}{cc}
s_{1} & s_{12} \\
s_{12} & s_{2}
\end{array}\right)=\left(\begin{array}{ll}
1 & 0 \\
q & 1
\end{array}\right)\left(\begin{array}{ll}
s_{1} & 0 \\
0 & w
\end{array}\right)\left(\begin{array}{ll}
1 & q \\
0 & 1
\end{array}\right),
$$

for $q=s_{12} / s_{1}$ and $w=s_{2}-s_{12}^{2} / s_{1}=(\operatorname{det} S) / s_{1}$. This shows that $\tilde{Z}(S, \rho)$ is a periodic function of $q$, when $S$ is decomposed as above. Then the formula of Selberg and Chowla is:

$$
\begin{aligned}
\tilde{Z}(S, \rho)= & s_{1}^{-\rho} \zeta(2 \rho)+\pi^{1 / 2} w^{1 / 2-\rho} s_{1}^{-1 / 2} \Gamma(\rho-1 / 2) \Gamma(\rho)^{-1} \zeta(2 \rho-1) \\
+ & 2 \pi^{\rho} w^{1 / 4-1 / 2 \rho} s_{1}^{-1 / 4-1 / 2 \rho} \Gamma(\rho)^{-1} \\
& \sum_{\lambda \neq 0} e^{2 \pi i \lambda q} \sigma_{1-2 \rho}(\lambda) \mid \lambda^{\rho-1 / 2} K_{1 / 2-\rho}\left(2 \pi|\lambda|\left(w / s_{1}\right)^{1 / 2}\right) .
\end{aligned}
$$

Here

$$
\sigma_{k}(n)=\sum_{0<d \mid n} d^{k}
$$

and

$$
K_{\nu}(z)=\frac{1}{2} \int_{0}^{\infty} e^{-1 / 2 z\left(u+u^{-1}\right)} u^{\nu-1} d u, \text { for }|\operatorname{larg} z|<1 / 2 \pi .
$$

The latter is the modified Bessel function of the second kind. And $\Gamma(x)$ is the gamma function. In [16] formula (1.3) is generalized to arbitrary $n$ in $n-1$ ways. The main result of this paper (Theorem 2.1) is a generalization of formula (1.3) of Selberg and Chowla.

Now the Epstein zeta function is not the only Eisenstein series associated with $S L(n, \mathbf{R})$. Others considered in [10] and [15] are called Selberg zeta functions by Maass. Here we shall consider only the case of one complex variable $\rho$. This case was considered in Koecher [9] and the function is defined for an $n \times n$ positive definite symmetric matrix $S$ and a complex variable $\rho$ with $\operatorname{Re} \rho>1 / 2 n$ by

$$
\tilde{Z}_{n_{1}}(S, \rho)=\sum_{A}|S[A]|^{-\rho} \text {. }
$$

Here $n \geqslant n_{1}$ and $S[A]={ }^{t} A S A$, where ${ }^{t} A$ is the transpose of $A$. We denote the determinant of a matrix $U$ by $|U|$ and its absolute value by $\|U\|$. The sum is over a complete set of representatives for the equivalence relation $A \sim B$ defined on $n \times n_{1}$ rank $n_{1}$ integral matrices $A, B$ as follows:

$A \sim B$ if $A=B V$, for $V$ some $n_{1} \times n_{1}$ integral matrix with $|V|= \pm 1$.

The notation set up here will be used throughout the paper. In addition we shall use the notation $S^{(n)}$ for an $n \times n$ matrix and $A^{\left(n, n_{1}\right)}$ for an $n \times n_{1}$ matrix. 
For $n_{1}=1$ the function of (1.6) is Epstein's zeta function (1.1) clearly.

The functional equation and analytic continuation of (1.6) are given in [10] and [15] using an argument of Selberg with differential operators on the space of positive definite symmetric matrices. One shows easily that

$$
\widetilde{Z}_{n_{1}}(S, \rho)=Z_{n_{1}}(S, \rho) \prod_{i=0}^{n_{1}-1} \zeta(2 \rho-i),
$$

where one defines

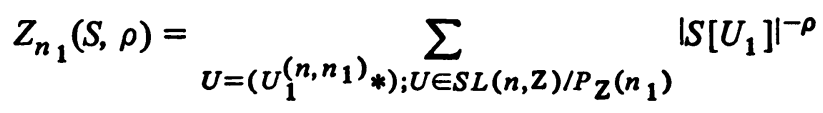

Here $P_{\mathrm{Z}}\left(n_{1}\right)$ is the subgroup of $\operatorname{SL}(n, \mathrm{Z})$ of matrices of block form

$$
\left(\begin{array}{cc}
A^{\left(n_{1}\right)} & B \\
0 & C
\end{array}\right)
$$

The notation means that the sum in (1.8) is over a complete set of representatives for the quotient $S L(n, \mathrm{Z}) / P_{\mathrm{Z}}\left(n_{1}\right)$. The residue of the pole of $\widetilde{Z}_{n_{1}}(S, \rho)$ at $\rho=$ $n / 2$ has been calculated [15]. And the other residues have reportedly been computed by Langlands (unpublished). It is hoped that Theorem 2.1 may ultimately shed some more light on the behavior of $\widetilde{Z}_{n_{1}}(S, \rho)$, just as the Selberg-Chowla formula (1.3) provides much information about Epstein's zeta function. For example, one might expect to be able to calculate the constant term in the Laurent expansion of $Z_{n_{1}}(S, \rho)$ about $\rho=n / 2$, thus generalizing Kronecker's limit formula.

Note that $Z_{n_{1}}(S, \rho)$ can be viewed as a function on $\operatorname{SL}(n, \mathrm{R})$ or on $S O(n) \backslash S L(n, \mathrm{R})$, where $S O(n)$ denotes the group of $n \times n$ orthogonal matrices of determinant one. This is achieved by setting $S=t_{g g}$ for $g \in S L(n, \mathbf{R})$. And $Z_{n_{1}}$ is an Eisenstein series in the sense of Borel [3]. Note that in particular, $Z_{n_{1}}$ is invariant under the transformation $S \rightarrow S[U]$, for $U$ integral $n \times n$ of determinant \pm 1 . The calculation of the Fourier coefficients for the Eisenstein series associated with the symplectic group has been done ([1], [8], [10], [11]). The main function arising in that calculation for the analytic case is a generalization of the arithmetical function $\sigma_{k}(n)$ of (1.4). Here we shall apply the method of Baily [1, pp. 228-240] to the special linear group. The functions arising in the Fourier coefficients will include not only the above arithmetical functions but also Bessel functions of matrix argument studied by Herz [5]. This is not surprising in view of the Selberg-Chowla formula as well as the results of Jacquet [6, pp. 283-285], Kaufhold [8], and Maass [10, pp. 300-313].

Thus the main result of the paper is the formula of Theorem 2.1 for the Fourier coefficients of $Z_{n_{1}}(S, \rho)$. The Fourier expansion is with respect to the 
Abelian subgroup of $\operatorname{SL}(n, \mathbf{R})$ of elements of the form

$$
A_{U}=\left(\begin{array}{cc}
I^{\left(n_{1}\right)} & U \\
0 & I
\end{array}\right),
$$

where $I$ is always the identity matrix and 0 is always the zero matrix. Note that $A_{U} A_{V}=A_{U+V}$ and that $Z_{n_{1}}(S, \rho)=Z\left(S\left[A_{U}\right], \rho\right)$ for integral matrices $U$. Thus $Z_{n_{1}}$ is a periodic function of $Q$, if we write $[13$, p. 24]

$$
S=\left(\begin{array}{cc}
S_{1}^{\left(n_{1}\right)} & 0 \\
0 & W
\end{array}\right)\left[\left(\begin{array}{cc}
I & Q \\
0 & I
\end{array}\right)\right] .
$$

The Fourier expansion of Theorem 2.1 is for this periodic function of $\mathbf{Q}$. The result is not as good as one might expect, for [16] would lead us to believe that more than one Fourier expansion for $Z_{n_{1}}$ exists. That is, one ought to be able to obtain expansions of $Z_{n_{1}}$ with respect to the subgroup of $\operatorname{SL}(n, \mathrm{R})$ of elements

$$
\left(\begin{array}{cc}
I^{(m)} & U \\
0 & I
\end{array}\right)
$$

for all $m$, with $1 \leqslant m<n$, and not just $m=n_{1}$. More general (e.g., nilpotent) Fourier analysis ought also to be considered.

We should note also that we always restrict our attention to $Z_{n_{1}}\left(S^{(n)}, \rho\right)$ with $2 n_{1} \leqslant n$. This is no real loss of generality in view of [15]

$$
Z_{n_{1}}\left(S^{(n)}, \rho\right)=|S|^{-\rho} Z_{n-n_{1}}\left(S^{-1}, \rho\right) .
$$

In the third part of the paper we study the Bessel functions of matrix argument arising in the Fourier coefficients of Theorem 2.1. The definition of these Bessel functions is slightly different from that of Herz [5]. The difference arose in the calculations of [16]. That the difference is only superficial is shown in Lemma 3.5. This lemma also gives an easy proof for formula (2.5) of [16]. The other results of $\S 3$ set up the necessary theory of Bessel functions which would be needed to use the Fourier expansion of Theorem 2.1 to obtain new information about the function $Z_{n_{1}}\left(S^{(n)}, \rho\right)$. For recall that (1.3) implies the Kronecker limit formula for the Epstein zeta function of a binary positive definite quadratic form (see [16]). Kaufhold [8] uses a formula analogous to Theorem 2.1 to obtain the analytic continuation and functional equation of the lowest dimensional nontrivial analogue of $Z_{n_{1}}\left(S^{(n)}, \rho\right)$ for the symplectic group. However it seems highly nontrivial to generalize Kaufhold's results to arbitrary dimension. Though some explicit formulas are to be found in [7] and [17]. 
2. The Fourier expansion. After a long series of lemmas we obtain a Fourier expansion of $Z_{n_{1}}\left(S^{(n)}, \rho\right), n \geqslant 2 n_{1}$, analogous to those obtained in [1], [8], [10], and [11] for Eisenstein series on the symplectic group. The method is the exact analogue of Baily's method [1, pp. 230-232].

Just as in (1.8), define

LEMMA 2.1. Let

$$
P_{\mathrm{Q}}\left(n_{1}\right)=\left\{\left(\begin{array}{cc}
A^{\left(n_{1}\right)} & B \\
0 & C
\end{array}\right) \in S L(n, Q)\right\} .
$$

$$
\sigma_{r}=\left(\begin{array}{cccc}
0 & 0 & I^{(r)} & 0 \\
0 & I^{\left(n_{1}-r\right)} & 0 & 0 \\
-I^{(r)} & 0 & 0 & 0 \\
0 & 0 & 0 & I^{\left(n-n_{1}-r\right)}
\end{array}\right)
$$

Then $\operatorname{SL}(n, \mathrm{Q})=\bigcup_{r=0}^{n_{1}} P_{\mathrm{Q}} \sigma_{r} P_{\mathrm{Q}}$, and the union is disjoint.

Proof. This formula can be proved by direct matrix calculation or one can imitate Baily's proof for the symplectic group using the Bruhat decomposition. Note that a matrix

$$
\left(\begin{array}{cc}
A^{\left(n_{1}\right)} & B \\
C & D
\end{array}\right) \in S L\left(n_{1}, Q\right)
$$

lies in the $r$ th set $P_{\mathrm{Q}} \sigma_{r} P_{\mathrm{Q}}$ if and only if the rank of $C$ is $r$.

Define the general linear group $G L(n, Q)$ to be the group of all $n \times n$ rational matrices with nonzero determinant and set

$$
\widetilde{P}_{\mathrm{Q}}\left(n_{1}\right)=\left\{\left(\begin{array}{cc}
A^{\left(n_{1}\right)} & B \\
0 & C
\end{array}\right) \in G L(n, \mathrm{Q})\right\} \text {. }
$$

LEMMA 2.2 .

$$
\begin{aligned}
& G L(n, \mathrm{Q})=\tilde{P}_{\mathrm{Q}}\left(n_{1}\right) \cdot S L(n, \mathrm{Z})=S L(n, \mathrm{Z}) \cdot \tilde{P}_{\mathrm{Q}}\left(n_{1}\right) . \\
& S L(n, \mathrm{Q})=P_{\mathrm{Q}}\left(n_{1}\right) \cdot S L(n, \mathrm{Z})=S L(n, \mathrm{Z}) \cdot P_{\mathrm{Q}}\left(n_{1}\right) .
\end{aligned}
$$

Proof. The proof is by induction on $n$. For given $A$ in $G L(n, \mathrm{Q})$, there exists a matrix $U$ in $S L(n, Z)$ such that $U A$ has the first $n-1$ elements in the last row equal to zero. For we know that $n-1$ equations in $n$ unknowns are solvable in $\mathbf{Q}$ and, if homogeneous, are solvable in $\mathbf{Z}$, with relatively prime coefficients. Treat the remaining $(n-1) \times(n-1)$ block matrix in the upper left-hand corner of $U A$ by induction.

Note that the proof actually shows that $P_{\mathrm{Q}}\left(n_{1}\right)$ can be replaced by the 
group of Q-rational points of a minimal Q-parabolic subgroup.

LEMMA 2.3. For $1 \leqslant n_{1} \leqslant[1 / 2 n]$ and $r=1,2, \cdots, n_{1}$, $\left(S L(n, Z) \cap\left(P_{\mathrm{Q}}\left(n_{1}\right) \sigma_{r} P_{\mathrm{Q}}\left(n_{1}\right)\right)\right) / P_{\mathrm{Z}}\left(n_{1}\right)$ has a complete set of representatives of the form

$$
\left(\begin{array}{cc}
{ }^{t} A^{-1} & 0 \\
0 & B
\end{array}\right) N_{U} \sigma_{r} P_{A, B, U},
$$

where

$$
N_{U}=\left(\begin{array}{cccc}
I^{(r)} & 0 & U^{(r)} & 0 \\
0 & I^{\left(n_{1}-r\right)} & 0 & 0 \\
0 & 0 & I^{(r)} & 0 \\
0 & 0 & 0 & I^{\left(n-n_{1}-r\right)}
\end{array}\right),
$$

for $U$ a rational matrix. and $A$ lies in a complete set of representatives for $S L\left(n_{1}, Z\right) / P_{Z}(r)$. Similarly $B$ runs through a complete set of representatives for $S L\left(n-n_{1}, Z\right) / P_{Z}(r)$, and $P_{A, B, U} \in P_{Q}\left(n_{1}\right)$ is fixed once $A, B, U$ are given.

Proof. Using Lemma 2.2 we obtain a map

$$
P_{\mathrm{Q}} \rightarrow\left(S L(n, \mathrm{Z}) \cap\left(P_{\mathrm{Q}}\left(n_{1}\right) \sigma_{r} P_{\mathrm{Q}}\left(n_{1}\right)\right)\right) / P_{\mathrm{Z}}\left(n_{1}\right) \text {. }
$$

For given $P \in P_{\mathrm{Q}}\left(n_{1}\right)$, there exists $P^{\prime} \in P_{\mathrm{Q}}\left(n_{1}\right)$ such that $P \sigma_{r} P^{\prime} \in S L(n, \mathrm{Z})$. The map is easily seen to be well defined.

In order to complete the proof we require another lemma.

Lemma 2.4. If $P, P^{\prime} \in P_{\mathrm{Q}}\left(n_{1}\right)$, the equation $\sigma_{r} P^{\prime}=P \sigma_{r}$ is equivalent to

$$
P=\left(\begin{array}{cccc}
A^{(r)} & 0 & 0 & B \\
C & D^{\left(n_{1}-r\right)} & E & F \\
0 & 0 & G^{(r)} & H \\
0 & 0 & 0 & K^{\left(n-n_{1}-r\right)}
\end{array}\right),
$$

with $P^{\prime}=\sigma_{r} P \sigma_{r}^{-1}$.

Proof. Multiply out the indicated matrices in the equation $\sigma_{r} P^{\prime}=P \sigma_{r}$.

We now return to the proof of Lemma 1.4. Note that $P \sigma_{r} P_{1}=P^{\prime} \sigma_{r} P_{1}^{\prime} \gamma$, for $P, P^{\prime}, P_{1}, P_{1}^{\prime} \in P_{Q}\left(n_{1}\right)$ and $\gamma \in P_{Z}\left(n_{1}\right)$ implies $P^{-1} P^{\prime} \sigma_{r}=\sigma_{r} P_{1} \gamma^{-1}\left(P_{1}^{\prime}\right)^{-1}$. Then Lemma 2.4 shows that

$$
P^{-1} P^{\prime}=\left(\begin{array}{cccc}
R & 0 & 0 & S \\
T & U & V & W \\
0 & 0 & X & Y \\
0 & 0 & 0 & Z
\end{array}\right)
$$


Now let

$$
P_{\mathbf{Q}}^{*}=\left\{\left(\begin{array}{cccc}
R & 0 & 0 & S \\
T & U & V & W \\
0 & 0 & X & Y \\
0 & 0 & 0 & Z
\end{array}\right) \in S L(n, Q)\right\}
$$

where the blocks have the usual sizes. We seek a complete system of representatives for $P_{\mathrm{Q}}\left(n_{1}\right) / P_{\mathrm{Q}}^{*}$. Using Lemma 2.2 one sees that for every $P \in P_{\mathrm{Q}}\left(n_{1}\right)$ there exists $P^{*} \in P_{\mathrm{Q}}^{*}$ such that

$$
P P^{*}=\left(\begin{array}{cc}
{ }^{t} A^{-1} & { }^{t} A^{-1} C \\
0 & B
\end{array}\right),
$$

for $A \in S L\left(n_{1}, \mathrm{Z}\right)$ and $B \in S L\left(n-n_{1}, \mathrm{Z}\right)$. Then we can reduce $A$ modulo $P_{\mathrm{Z}}(r)$ and $B$ modulo $P_{\mathrm{Z}}(r)$. Finally one obtains $C$ of the form

$$
\left(\begin{array}{ll}
U^{(r)} & 0 \\
0 & 0
\end{array}\right)
$$

LEMMA 2.5. Suppose that

$$
\left(\begin{array}{cc}
{ }^{t} A^{-1} & 0 \\
0 & B
\end{array}\right) N_{U} \sigma_{r} P_{A, B, U} \in S L(n, Z),
$$

where $A, B, U$ are as in Lemma 2.3 and

$$
P_{A, B, U}=\left(\begin{array}{cc}
P_{1}^{\left(n_{1}\right)} & * \\
0 & *
\end{array}\right) \in P_{\mathrm{Q}}\left(n_{1}\right) .
$$

Then $\left\|P_{1}\right\|=\nu(U)$, where we define $\nu(U)=$ the product of the reduced denominators of the elementary divisors of $U$.

Proof. Clearly it is equivalent to ask that $N_{U} \sigma_{r} P$ lie in $S L(n, \mathrm{Z})$. Suppose now that

$$
P_{1}=\left(\begin{array}{cc}
E^{(r)} & F \\
G & H
\end{array}\right)
$$

Then

$$
N_{U} \sigma_{r} P=\left(\begin{array}{cccc}
-U E & -U F & * & * \\
G & H & * & * \\
-E & -F & * & * \\
0 & 0 & * & *
\end{array}\right) \text {. }
$$

By elementary divisor theory assume that 


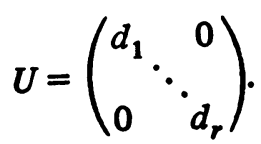

This changes $P_{1}$ but not its determinant. Then the $i$ th row of $U(E F)$ is $d_{i}$ times the $i$ th row of $(E F)$. Suppose $q_{i}$ is the reduced denominator of $d_{i}$. Clearly $q_{1} q_{2} \cdots q_{r}$ divides $\left|P_{1}\right|$. If some integer $m$ divides $\left(q_{1} q_{2} \cdots q_{r}\right)^{-1}\left|P_{1}\right|$, then $m$ divides $1=\left|N_{U} \sigma_{r} P\right|$, because $m$ divides all terms in the $n_{1} \times n_{1}$ block Lagrange decomposition of $W_{U} \sigma_{r} P \mid$ corresponding to expansion by the first $n_{1}$ columns. For each term contains an $n_{1} \times n_{1}$ subdeterminant from the first $n_{1}$ columns of $N_{U} \sigma_{r} P$. Such $n_{1} \times n_{1}$ subdeterminants are either 0 or a product of some $d_{i}$ times $\left|P_{1}\right|$.

We are now in a position to rearrange the terms of $Z_{n_{1}}\left(S^{(n)}, \rho\right)$ for $n \geqslant 2 n_{1}$ in such a way as to apply the Poisson summation formula.

First we make a definition. Suppose that

$$
\begin{aligned}
& S=\left(\begin{array}{cc}
S_{1}^{\left(n_{1}\right)} & 0 \\
0 & W^{\left(n-n_{1}\right)}
\end{array}\right)\left[\left(\begin{array}{ll}
I & Q \\
0 & I
\end{array}\right)\right], \\
& Q=\left(\begin{array}{ll}
Q_{r}^{(r)} & * \\
Q_{r}^{\prime} & *
\end{array}\right), \quad W=\left(\begin{array}{ll}
W_{r}^{(r)} & * \\
& *
\end{array}\right) \text {, } \\
& S_{1}=\left(\begin{array}{cc}
T_{r}^{(r)} & 0 \\
0 & { }_{r} S^{\left(n_{1}-r\right)}
\end{array}\right)\left[\left(\begin{array}{cc}
I & 0 \\
P & I
\end{array}\right)\right] .
\end{aligned}
$$

Then define

$$
\psi_{r}(S, \rho)=\left.\left.\right|_{r} S\right|^{-\rho} \sum_{U \in M(r, z)}\left|T_{r}\left[Q_{r}+U\right]+W_{r}\right|^{-\rho} .
$$

Here $M(r, \mathrm{Z})$ denotes the set of all $r \times r$ matrices with integral entries.

In the following lemma we shall, as usual, write $\Sigma_{x \in G / H} f(x)$ to mean that the sum runs over a complete set of representatives $x$ in $G$ for the quotient space $G / H$ when $f: G \rightarrow C$ is constant on cosets of $H$.

$$
\begin{aligned}
& \text { LEMMA 2.6. } \\
& Z_{n_{1}}\left(S^{(n)}, \rho\right)=\left|S_{1}\right|^{-\rho}+\sum_{1<r<n_{1} ; A, B, R} v(R)^{-2 \rho} \psi_{r}\left(S\left[\left(\begin{array}{cc}
t^{-1} & 0 \\
0 & B
\end{array}\right) N_{R}\right], \rho\right)
\end{aligned}
$$

where $A \in S L\left(n_{1}, Z\right) / P_{Z}(r), B \in S L\left(n-n_{1}, Z\right) / P_{Z}(r)$ (multiplicative quotients) and $R \in M(r, \mathrm{Q}) / M(r, \mathrm{Z})$ (additive quotient). As usual, we assume $n \geqslant 2 n_{1}$ and $\operatorname{Re} \rho>1 / 2 n$. 
Proof. By Lemma 2.5 and (2.1), if

$$
P=\left(\begin{array}{cc}
P_{1}^{\left(n_{1}\right)} & * \\
0 & *
\end{array}\right) \in P_{\mathrm{Q}}\left(n_{1}\right)
$$

is such that $N_{U} \sigma_{r} P \in S L(n, Z)$ then

$$
\left|S\left[N_{U} \sigma_{r}\left(\begin{array}{c}
P_{1}^{(r)} \\
0
\end{array}\right)\right]\right|=\left.\nu(U)^{2}\left|T_{r}\left[U+Q_{r}\right]+W_{r}\right| \cdot\right|_{r} S \mid .
$$

The proof of this fact runs as follows.

$$
\begin{aligned}
\left|S\left[\left(\begin{array}{cc}
U & 0 \\
0 & -I \\
I & 0 \\
0 & 0
\end{array}\right)\right]\right| & =\left|\left(\begin{array}{cc}
S_{1} & 0 \\
0 & W
\end{array}\right)\left[\left(\begin{array}{cccc}
I & 0 & Q_{r} & * \\
0 & I & Q_{r}^{\prime} & * \\
0 & 0 & I & 0 \\
0 & 0 & 0 & I
\end{array}\right)\left(\begin{array}{cc}
U & 0 \\
0 & -I \\
I & 0 \\
0 & 0
\end{array}\right)\right]\right| \\
& =\left|S_{1}\left[\left(\begin{array}{cc}
U+Q_{r} & 0 \\
Q_{r}^{\prime} & -I
\end{array}\right)\right]+\left(\begin{array}{cc}
W_{r} & 0 \\
0 & 0
\end{array}\right)\right| \\
& =\left|S_{1}\left[\left(\begin{array}{cc}
U+Q_{r} & 0 \\
0 & I
\end{array}\right)\right]+\left(\begin{array}{cc}
W_{r} & 0 \\
0 & 0
\end{array}\right)\right|,
\end{aligned}
$$

since

and

$$
\left(\begin{array}{cc}
U+Q_{r} & 0 \\
Q_{r}^{\prime} & -I
\end{array}\right)=\left(\begin{array}{cc}
U+Q_{r} & 0 \\
0 & I
\end{array}\right)\left(\begin{array}{cc}
I & 0 \\
Q_{r}^{\prime} & -I
\end{array}\right)
$$

$$
\left(\begin{array}{ll}
W_{r} & 0 \\
0 & 0
\end{array}\right) \quad\left(\begin{array}{ll}
W_{r} & 0 \\
0 & 0
\end{array}\right)\left(\begin{array}{cc}
I & 0 \\
Q_{r}^{\prime} & -I
\end{array}\right) .
$$

In a similar way one obtains the further simplification

$$
\begin{aligned}
\left|S_{1}\left[\left(\begin{array}{cc}
U+Q_{r} & 0 \\
0 & I
\end{array}\right)\right]+\left(\begin{array}{ll}
W_{r} & 0 \\
0 & 0
\end{array}\right)\right| \\
=\left|\left(\begin{array}{cc}
T_{r} & 0 \\
0 &
\end{array}\right)\left[\left(\begin{array}{cc}
U+Q_{r} & 0 \\
P\left(U+Q_{r}\right) & I
\end{array}\right)\right]+\left(\begin{array}{cc}
W_{r} & 0 \\
0 & 0
\end{array}\right)\right| \\
=\left.\left|T_{r}\left[U+Q_{r}\right]+W_{r}\right|\right|_{r} S \mid .
\end{aligned}
$$


An application of Lemma 2.3 completes the proof.

For future use it would be well to consider $\psi_{r}(\tilde{S}, \rho)$ a little more closely, where

$$
\tilde{S}=S\left[\left(\begin{array}{cc}
A^{-1} & 0 \\
0 & B
\end{array}\right) N_{R}\right] .
$$

Using formula (2.1) we obtain

$$
\begin{aligned}
\left(\begin{array}{ll}
S_{1} & 0 \\
0 & W
\end{array}\right)\left[\left(\begin{array}{ll}
I & Q \\
0 & I
\end{array}\right)\left(\begin{array}{cc}
{ }^{t} A^{-1} & 0 \\
0 & B
\end{array}\right)\left(\begin{array}{cc}
I & \left(\begin{array}{cc}
R & 0 \\
0 & 0
\end{array}\right) \\
0 & I
\end{array}\right)\right] \\
=\left(\begin{array}{cc}
\left.S_{1}{ }^{t} A^{-1}\right] & 0 \\
0 & W[B]
\end{array}\right)\left[\left(\begin{array}{cc}
I & { }^{t} A Q B+\left(\begin{array}{ll}
R & 0 \\
0 & 0
\end{array}\right) \\
0 & I
\end{array}\right)\right]
\end{aligned}
$$

Note also that

$$
S_{1}^{-1}=\left(\begin{array}{cc}
T_{r}^{-1} & 0 \\
0 & { }_{r} S^{-1}
\end{array}\right)\left[\left(\begin{array}{cc}
I & -{ }^{t} P \\
0 & I
\end{array}\right)\right]
$$

It follows that if

$$
S_{1}^{-1}=\left(\begin{array}{cc}
\left(S_{1}^{-1}\right)_{r} & * \\
* & *
\end{array}\right)
$$

then $\left(S_{1}^{-1}\right)_{r}=T_{r}^{-1}$. Applying this to the decomposition

$$
S_{1}\left[{ }^{t} A^{-1}\right]=\left(\begin{array}{cc}
T_{r}^{A} & 0 \\
0 & { }^{A}
\end{array}\right)\left[\left(\begin{array}{cc}
I & 0 \\
P^{A} & I
\end{array}\right)\right],
$$

we see that $T_{r}^{A}=\left(S_{1}^{-1}\left[A_{r}\right]\right)^{-1}$, where $A^{\left(n_{1}\right)}=\left(A_{r}^{\left(n_{1}, r\right)} *\right)$. Also $\left.\right|_{r} S^{A} \mid=$ $\left|S_{1}\right|\left|T_{r}^{A}\right|^{-1}=\left|S_{1}\right| S_{1}^{-1}\left[A_{r}\right] \mid$. We have thus proved the following lemma.

LEMMA 2.7.

$$
\begin{aligned}
Z_{n_{1}}\left(S^{(n),}, \rho\right)= & \left|S_{1}\right|^{-\rho} \\
& +\left|S_{1}\right|^{-\rho} \sum_{A, B, U, R ; 1<r<n_{1}} \nu(R)^{-2 \rho}\left|S_{1}^{-1}\left[A_{r}\right]\right|^{-\rho} \\
& \times \mid\left(S_{1}^{-1}\left[A_{r}\right)^{-1}\left[{ }^{t} A_{r} Q B_{r}+R+U\right]+\left.W\left[B_{r}\right]\right|^{-\rho}\right.
\end{aligned}
$$

where 


$$
\begin{aligned}
& A=\left(A_{r}^{\left(n_{1}, r\right)} *\right) \in S L\left(n_{1}, \mathrm{Z}\right) / P_{\mathrm{Z}}(r), \\
& B=\left(B_{r}^{\left(n-n_{1}, r\right)} *\right) \in S L\left(n-n_{1}, \mathrm{Z}\right) / P_{\mathrm{Z}}(r), \\
& U \in M(r, \mathrm{Z}), \quad R \in M(r, \mathbf{Q}) / M(r, \mathrm{Z}) .
\end{aligned}
$$

And $\operatorname{Re} \rho>1 / 2 n, n \geqslant 2 n_{1}$.

It is not perhaps obvious that this is the same as the simple decomposition used in Theorem 1 of [16] for $\widetilde{Z}_{1}\left(S^{(n)}, \rho\right)$. There we obtained

$$
\zeta(2 \rho) Z_{1}\left(S^{(n)}, \rho\right)=\zeta(2 \rho) s_{1}^{-\rho}+\frac{1}{2} \sum_{a \in Z ; b \in Z^{n-1-0}}\left(s_{1}[a+Q b]+W[b]\right)^{-\rho} .
$$

In this case $n_{1}=1$ and Lemma 2.8 gives

$$
\zeta(2 \rho) Z_{1}\left(S^{(n)}, \rho\right)
$$

$$
=\zeta(2 \rho)\left\{s_{1}^{-\rho}+\sum_{\left(b_{1} *\right), u, r} v(r)^{-2 \rho}\left(s_{1}\left[Q b_{1}+r+u\right]+W\left[b_{1}\right]^{-\rho}\right\}\right.
$$

where $\left(b_{1} *\right) \in S L(n-1, Z) / P_{Z}(1), u \in Z, r \in Q / Z$. The last sum equals

$$
\frac{1}{2} \sum\left(s_{1}\left[Q b_{1} q a+p a\right]+W\left[b_{1} q a\right]\right)^{-\rho} \text {, }
$$

where the sum is over $b_{1} \in Z^{n-1}$ with relatively prime entries, $p / q \in \mathbf{Q}$ with $(p, q)=1, q>0$, and $a>0$. To see that the 2 decompositions are equivalent, note that

$\left\{\left(b_{1} q a, p a\right) \mid b_{1} \in Z^{n-1}\right.$ such that

$$
\begin{aligned}
& \text { g.c.d. } \left.\left(b_{1}\right)=1, p / q \in \mathbf{Q} \text { with }(p, q)=1, q>0, a>0\right\} \\
& =\left\{(X, y) \mid X \in \mathbf{Z}^{n-1}-0, y \in \mathbf{Z}\right\} .
\end{aligned}
$$

To go from $(X, y)$ to $\left(b_{1} q a, p a\right)$ set $a=$ g.c.d. $(X, y)$ and $q=$ g.c.d. $\left(a^{-1} X\right)$.

One should note that the decomposition we used in [16, Theorem 1] was more general. Thus one would conjecture that a more general decomposition can be obtained for $Z_{n_{1}}\left(S^{(n)}, \rho\right)$.

We can rearrange the series as in the preceding lemmas because the series $Z_{n_{1}}\left(S^{(n)}, \rho\right)$ converges absolutely and uniformly in compacta for $\operatorname{Re} \rho>1 / 2 n$ and $S$ positive definite symmetric.

The function $\psi_{r}(S, \rho)$ of (2.2) is invariant under $Q_{r} \rightarrow Q_{r}+U$ with $U \in$ $M(r, \mathrm{Z})$ and $Q_{r}$ as in (2.1). Thus it has a Fourier expansion (for $\operatorname{Re} \rho>1 / 2 n$ )

$$
\psi_{r}(S, \rho)=\sum_{N \in M(r, z)} a_{N}(S) \exp \left\{2 \pi i \sigma\left({ }^{t} N Q_{r}\right)\right\},
$$

where $\sigma(X)=$ trace of $X$. 
Before evaluating the Fourier coefficients $a_{N}(S)$ we need a few definitions. For $A, B$ both $n \times r$ real matrices define the modified Bessel function of the second kind $K(A, B, \rho)$ by

$$
K(A, B, \rho)=\int_{t_{X=X>0}} \mid X^{\rho-1 / 2(r+1)} \exp \left\{-\sigma\left(t^{t} A A X+{ }^{t} B B X^{-1}\right)\right\} d X
$$

Here $d X=$ Lebesgue measure on $\mathrm{R}^{1 / 2 n(n+1)}$. This function was considered by Herz [5] essentially and will be discussed more fully in the next section. In the case $n=r=1, z>0, K(\sqrt{z}, \sqrt{z}, \rho)=2 K_{\rho}(2 z)$, where $K_{\rho}(z)$ was defined in (1.5). The function (2.6) converges for all $\rho$ if ${ }^{t} A A$ and ${ }^{t} B B$ are both positive definite. If ${ }^{t} A A$ is positive definite and ${ }^{t} B B$ is not, then conditions on $\rho$ such as $\operatorname{Re} \rho>1 / 2(r-1)$ are needed $(n \geqslant r)$. Note that $[1$, p. 226]

$$
K\left(I^{(n)}, 0, \rho\right)=\pi^{1 / 4 n(n-1)} G_{n}(\rho),
$$

where $G_{n}(\rho)=\Gamma(\rho) \Gamma(\rho-1 / 2) \cdots \Gamma(\rho-1 / 2(n-1))$.

When $S$ is positive definite symmetric, we shall write $T=\sqrt{S}$ to mean a matrix $T \in G L(n, \mathrm{R})$ such that ${ }^{t} T T=S$.

Finally we define

$$
\sigma(U, \rho)=\sum_{R \in M(r, \mathrm{Q}) / M(r, \mathrm{Z})} \nu(R)^{-2 \rho} \exp \left\{2 \pi i \sigma\left({ }^{t} R U\right)\right\} .
$$

This is the exact analogue of the function $S$ which appears in Siegel's calculation of the Fourier coefficient of the Eisenstein series on the symplectic group [11, p. 133]. Note that our sum is over $r \times r$ rational matrices $R$ modulo 1 , while Siegel's sum is more restricted, being only over $r \times r$ symmetric rational matrices modulo 1. A slight modification of the arguments of [12, Lemmas 9, 10, and 11] shows that $\sigma(U, \rho)$ converges for $\operatorname{Re} \rho>r$. The main change in Siegel's argument is to replace the exponent $n-k+1$ in formula (22) of [12] by $n$, because of the lack of symmetry in the case under consideration.

THEOREM 2.1. For $\operatorname{Re} \rho>1 / 2 n$ and $1 \leqslant n_{1} \leqslant 1 / 2 n$,

$$
\begin{aligned}
Z_{n_{1}}\left(S^{(n)}, \rho\right)= & \left|S_{1}\right|^{-\rho} \\
+ & \left|S_{1}\right|^{-\rho} \sum_{r=1}^{n_{1}} \pi^{1 / 4\left(r^{2}+r\right)} G_{r}(\rho)^{-1} \\
& \times \sum_{A, B, U} \sigma(U, \rho)\left(\left|S_{1}^{-1}\left[A_{r}\right]\right| W\left[B_{r}\right] \mid\right)^{1 / 2 r-\rho} \\
& \times \exp \left\{2 \pi i \sigma\left({ }^{t} U^{t} A_{r} Q B_{r}\right)\right\} K\left(I, \pi \sqrt{S_{1}^{-1}\left[A_{r}\right]} U \sqrt{W\left[B_{r}\right]}, \rho-1 / 2 r\right) .
\end{aligned}
$$

Here $A \in S L\left(n_{1}, \mathrm{Z}\right) / P_{\mathrm{Z}}(r), B \in S L\left(n-n_{1}, \mathrm{Z}\right) / P_{\mathrm{Z}}(r), U \in M(r, \mathrm{Z})$. 
Proof. From (2.5) and (2.2) we see that

$$
a_{U}(S)=\left.\left.\int_{M(r, \mathrm{R})}\left|T_{r}\left[Q_{r}+X\right]+W_{r}\right|^{-\rho}\right|_{r} S\right|^{-\rho} \exp \left\{-2 \pi i \sigma\left({ }^{t} U X\right)\right\} d X .
$$

Here $d X$ is Lebesgue measure on $\mathbf{R}^{r^{2}}$. Making the change of variables $Y=$ $\sqrt{T_{r}}\left(Q_{r}+X\right)$, we obtain

$$
\begin{aligned}
a_{U}(S)= & \left.\left.\left|T_{r}\right|^{-1 / 2 r}\right|_{r} S\right|^{-\rho} \exp \left\{2 \pi i \sigma\left({ }^{t} U Q_{r}\right)\right\} \\
& \times \int_{M(r, R)}\left|{ }^{t} T T+W_{r}\right|^{-\rho} \exp \left\{-2 \pi i \sigma\left({ }^{t} U\left(\sqrt{T_{r}}\right)^{-1} Y\right)\right\} d Y .
\end{aligned}
$$

Note that since $T_{r}={ }^{t}\left(\sqrt{T_{r}}\right) \sqrt{T_{r}}, T_{r}^{-1}=\left(\sqrt{T_{r}}\right)^{-1} t\left(\sqrt{T_{r}}\right)^{-1}$. Thus $\sqrt[t]{T_{r}^{-1}}=$ $O\left(\sqrt{T_{r}}\right)^{-1}$ for some orthogonal matrix $O$. A change of variables then shows that we may replace $\exp \left\{-2 \pi i \sigma\left({ }^{t} U\left(\sqrt{T_{r}}\right)^{-1} Y\right)\right\}$ inside the integral by

$$
\exp \left\{2 \pi i \sigma\left(t\left(\sqrt{T_{r}^{-1}} U\right) Y\right)\right\} \text {. }
$$

From Lemmas 3.1 and 3.3 of the next section it is seen that

$$
\begin{aligned}
a_{U}(S)= & \left.\left.\left|T_{r}\right|^{-1 / 2 r}\right|_{r} S\right|^{-\rho} \exp \left\{2 \pi i \sigma\left({ }^{t} U Q_{r}\right)\right\} \pi^{1 / 4\left(r^{2}+r\right)} \\
& \times G_{r}(\rho)^{-1} K\left(\sqrt{W_{r}}, \sqrt{T_{r}^{-1}} U, \rho-1 / 2 r\right) \\
= & \left.\left.\exp \left\{2 \pi i \sigma\left({ }^{t} U Q_{r}\right)\right\}\left|T_{r}\right|^{-1 / 2 r}\right|_{r} S\right|^{-\rho}\left|W_{r}\right|^{-(\rho-1 / 2 r)} \pi^{1 / 4\left(r^{2}+r\right)} \\
& \times G_{r}(\rho)^{-1} K\left(I, \sqrt{T_{r}^{-1}} U^{t} \sqrt{W_{r}}, \rho-1 / 2 r\right) .
\end{aligned}
$$

Due to the calculations preceding Lemma 2.7, we must substitute

$$
\begin{aligned}
& { }^{t} A_{r} Q B_{r}+R \text { for } Q_{r}, \quad\left(S_{1}^{-1}\left[A_{r}\right]\right)^{-1} \text { for } T_{r}, \\
& \left|S_{1}\right|\left|S_{1}^{-1}\left[A_{r}\right]\right| \text { for }\left.\right|_{r} S \mid, \quad W\left[B_{r}\right] \text { for } W_{r},
\end{aligned}
$$

into the formula for $a_{U}(S)$ to obtain

$$
\begin{aligned}
a_{U}\left(S\left[\left(\begin{array}{cc}
{ }^{t} A^{-1} & 0 \\
0 & B
\end{array}\right) N_{R}\right]\right) & \\
= & \left.\exp \left\{2 \pi i \sigma\left({ }^{t} U^{t} A_{r} Q B_{r}\right)\right\}\left|S_{1}^{-1}\left[A_{r}\right]^{1 / 2 r}\right| S_{1}\right|^{-\rho}\left|S_{1}^{-1}\left[A_{r}\right]\right|^{-\rho} \\
& \times \mid W\left[B_{r}\right]^{1 / 2 r-\rho} \pi^{1 / 4\left(r^{2}+r\right)} \exp \left\{2 \pi i \sigma\left({ }^{t} U R\right)\right\} G_{r}(\rho)^{-1} \\
& \times K\left(I, \sqrt{S_{1}^{-1}\left[A_{r}\right]} U^{t} \sqrt{W\left[B_{r}\right]}, \rho-1 / 2 r\right) .
\end{aligned}
$$

Thus, by Lemma 2.6, the proof is complete.

In the special case $n_{1}=1$, the result can be checked with Theorem 1 of [16]. The main formula needed in the comparison is 
where for $u=0$ we define

$$
\zeta(2 \rho) \sigma(u, \rho)=\sum_{0<d \mid u} d^{1-2 \rho}=\sigma_{1-2 \rho}(u),
$$

$$
\sigma_{1-2 \rho}(0)=\sum_{0<d \mid u} d^{1-2 \rho}=\zeta(2 \rho-1) .
$$

Ușing the formulas (1.5), (2.6), (2.7) as well, one sees that the formula of Theorem 2.1 yields:

$$
\begin{aligned}
& \tilde{Z}_{1}\left(S^{(n)}, \rho\right) \\
& \left.=\zeta(2 \rho) s_{1}^{-\rho}+\pi^{1 / 2} s_{1}^{-1 / 2} \Gamma^{\prime} \rho-1 / 2\right) \Gamma(\rho)^{-1} \tilde{Z}_{1}\left(W^{(n-1)}, \rho-1 / 2\right) \\
& +s_{1}^{-1 / 4-1 / 2 \rho} \Gamma(\rho)^{-1} \pi^{\rho} \sum_{\text {g.c.d. }\left(b_{1}\right)=1 ; u \in Z-\{0\}} \sum_{0<d \mid u} d^{1-2 \rho}\left(W\left[b_{1}\right] u^{-2}\right)^{1 / 4-1 / 2 \rho} \\
& \quad \times \exp \left\{2 \pi i u Q b_{1}\right\} K_{\rho-1 / 2}\left(2 \pi s_{1}^{-1 / 2}|u| \sqrt{W\left[b_{1}\right]}\right) \\
& =\zeta(2 \rho) s_{1}^{-\rho}+\pi^{1 / 2} s_{1}^{-1 / 2} \Gamma(\rho-1 / 2) \Gamma(\rho)^{-1} \tilde{Z}_{1}\left(W^{(n-1)}, \rho-1 / 2\right) \\
& +\left.s^{-1 / 4-1 / 2 \rho} \Gamma(\rho)^{-1} \pi^{\rho} \quad \sum_{n \in Z-\{0\} ; b \in Z^{n-1}-\{0\}} \ln \right|^{\rho-1 / 2}(W[b])^{1 / 4-1 / 2 \rho} \\
& \quad \times e^{2 \pi \ln Q b} K_{\rho-1 / 2}\left(2 \pi s_{1}^{-1 / 2}|n| \sqrt{W[b]}\right)
\end{aligned}
$$

upon setting $u=d n$ and then replacing $b_{1}$ by $d b_{1}$. This is the result of [16, Theorem 1] in the particular case corresponding to the $(1, n-1)$ decomposition with a minor change in point of view.

3. Bessel functions of matrix argument. We saw in the preceding section that certain modified Bessel functions of the second kind arise in the coefficients of Eisenstein series on $\operatorname{SL}(n, \mathrm{R})$. In this section we shall consider these functions carefully. Our definitions differ slightly from those of Herz [5] in order to derive an easy proof of a fact needed in [16] (see Lemma 3.5).

For $A$ and $B$ both $n \times r$ real matrices we define

$$
K(A, B, \rho)=\int_{t_{X}=X(r)>0}|X|^{\rho-1 / 2(r+1)} \exp \left\{-\sigma\left({ }^{t} A A X+{ }^{t} B B X^{-1}\right)\right\} d X
$$

$$
K_{1}(A, B, \rho)=\int_{W \text { real } n \times r}\left|{ }^{t} A A+{ }^{t} W W\right|^{-\rho} \exp \left\{2 i \sigma\left({ }^{t} B W\right)\right\} d W .
$$

The convergence, symmetry and reduction properties of these functions are considered in the following lemmas.

LEMMA 3.1. (a) Let $C^{(r)}$ be an invertible matrix. Then $K(A, B, \rho)=$ $K\left(A^{t} C, B C^{-1}, \rho\right)|C|^{2 \rho}$. 
(b) $K(A, B, \rho)=K(B, A,-\rho)$.

(c) For all orthogonal matrices $U, V, K(U A, V B, \rho)=K(A, B, \rho)$.

Proof. (a) Change variables via $X=Y[C]$.

(b) Change variables via $X=Y^{-1}$.

(c) Note that $I[U A]=I[A]$.

LEMMA 3.2. $K(A, B, \rho)$ converges for all $\rho$ if ${ }^{t} A A>0$ and ${ }^{t} B B>0$.

Proof. Change variables via $X={ }^{t} T T$, where

$$
T=\left(\begin{array}{ll}
t_{1} & \\
& t_{i j} \\
0 & t_{n}
\end{array}\right), \quad t_{i}>0 .
$$

Then

$$
K(A, B, \rho)=2^{r} \int_{t_{i}>0 ; t_{i j} \in \mathrm{R}} \prod_{i=1}^{r} t_{i}^{2 \rho-i} \exp \left\{-\sigma\left(I\left[A^{t} T\right]+I\left[B T^{-1} D\right\} d T .\right.\right.
$$

Here $d T$ denotes the ordinary Euclidean measure in $R^{1 / 2 r(r+1)}$.

Now, since ${ }^{t} A A$ and ${ }^{t} B B$ are both positive, there exist positive constants $c_{A}$ and $c_{B}$ depending only on $A$ and $B$ such that

$$
\sigma\left(I\left[A^{t} T\right] \geqslant c_{A} \sigma(t T) \text { and } \sigma\left(I\left[B T^{-1}\right]\right) \geqslant c_{B} \sigma\left(T^{-1} T^{-1}\right):\right.
$$

Therefore, with some positive constant $c$, depending only on $A$ and $B$, we have:

$$
\begin{aligned}
K(A, B, \rho)<c^{-1} \prod_{i=1}^{r} & \int_{0}^{\infty} t_{i}^{2 \rho-i} \exp \left\{-c t_{i}^{2}-c t_{i}^{-2}\right\} d t_{i} \\
& \times \prod_{i<j} \int_{-\infty}^{+\infty} \exp \left\{-c t_{i j}^{2}-c t_{i j}^{* 2}\right\} d t_{i j},
\end{aligned}
$$

where $T^{-1}=\left(t_{i j}^{*}\right)$.

The above integrals converge for all values of $\rho$, for

$$
\begin{aligned}
\int_{0}^{\infty} t^{a} \exp \left\{-c\left(t^{2}+t^{-2}\right)\right\} \frac{d t}{t}=\int_{1}^{\infty} t^{-a} \exp \left\{-c\left(t^{2}+t^{-2}\right)\right\} \frac{d t}{t} \\
+\int_{1}^{\infty} t^{a} \exp \left\{-c\left(t^{2}+t^{-2}\right)\right\} \frac{d t}{t}
\end{aligned}
$$

And these integrals converge for all values of $a$, since $a$ can be replaced by any larger value of $a$ obtaining an integral bounded by $\Gamma(1+|a|)$ for example.

We next prove the result needed in the proof of Theorem 1 connecting $K$ and $K_{1}$. 
LEMMA 3.3. $\pi^{3 / 4\left(r^{2}-r-2 r n\right)} G_{r}(\rho) K_{1}(A, B, \rho)=K(A, B, \rho-1 / 2 n)$, where $G_{r}(\rho)$ is the gamma factor defined by (2.7).

Proof. By (2.7)

$$
\begin{aligned}
& \pi^{3 / 4 r(r-1)} G_{r}(\rho) K_{1}(A, B, \rho) \\
& =\int_{W(n, r) \text { real }} \int_{X(r)>0} \exp \left\{-\sigma\left(\left({ }^{t} A A+{ }^{t} W W\right) X\right)+2 i \sigma\left({ }^{t} B W\right)\right\} \\
& \times|X|^{\rho-(r+1) / 2} d X d W \\
& =\int_{X>0}|X|^{\rho-1 / 2(r+1)} \exp \left\{-\sigma\left({ }^{t} A A X\right)\right\} \\
& \times \int_{W(n, r)} \text { real } \exp \left\{-\sigma\left({ }^{t} W W X+2 i^{t} B W\right)\right\} d W d X .
\end{aligned}
$$

The inner integral over $W$ is equal to

$$
|X|^{-1 / 2 n} \int_{W^{\prime}>0} \exp \left\{-\sigma\left(W^{\prime} W^{\prime}+2 i^{t} B W^{\prime} Z^{-1}\right)\right\} d W^{\prime},
$$

where $W^{\prime}=W Z, X=Z^{t} Z$.

Setting ${ }^{t} C=i Z^{-1} t_{B}$, we see that $\sigma\left({ }^{t} W^{\prime} W^{\prime}+2 i^{t} B W^{\prime} Z^{-1}\right)=\sigma\left(I\left[W^{\prime}+C\right]\right)+$ $\sigma\left({ }^{t} B B X^{-1}\right)$. Thus, using the formula [1, p. 244],

$$
\int_{W(n, r)_{\text {;real }}} \exp \left\{-\sigma(I[W+C D)\} d W=\int_{W(n, r)_{\text {real }}} \exp \{-\sigma(W W)\} d W=\pi^{1 / 2 n r}\right.
$$

and the proof is complete.

The question of the behavior of $K(I, B, \rho)$ for singular $B$ is answered by the following lemma.

LEMMA 3.4.

$$
K\left(I^{(r)},\left(\begin{array}{cc}
0 & 0 \\
0 & D^{(r-s)}
\end{array}\right), \rho\right)=\pi^{3 / 4 s(2 r-s-1)} G_{s}(\rho) K\left(I^{(r-s)}, D, \rho-1 / 2 s\right) .
$$

Proof. Write

$$
X=\left(\begin{array}{cc}
X_{1}^{(s)} & 0 \\
0 & W
\end{array}\right)\left[\left(\begin{array}{cc}
I & Q \\
0 & . I
\end{array}\right)\right]=\left(\begin{array}{cc}
X_{1} & X_{1} Q \\
t Q X_{1} & W+X_{1}[Q]
\end{array}\right)
$$

so that

$$
X^{-1}=\left(\begin{array}{cc}
X_{1}^{-1} & 0 \\
0 & W^{-1}
\end{array}\right)\left[\left(\begin{array}{cc}
I & 0 \\
-Q & I
\end{array}\right)\right]=\left(\begin{array}{cc}
X_{1}^{-1}+W^{-1}[Q] & -{ }^{t} Q W^{-1} \\
-W^{-1} Q & W^{-1}
\end{array}\right) .
$$

Then the Jacobian of the change of variables is $\left|\partial X / \partial\left(X_{1}, Q, W\right)\right|=\left|X_{1}\right|^{\mu-s}$. 
Thus

$$
\begin{aligned}
& K\left(I^{(r)},\left(\begin{array}{cc}
0 & 0 \\
0 & D^{(r-s)}
\end{array}\right), \rho\right) \\
& =\int_{X(s)>0 ; W(r-s)>0 ;} \exp \left\{-\sigma\left(X_{1}+W+X_{1}[Q]\right)-\sigma\left(D W^{-1}\right)\right\} \\
& Q^{(s, r-s)} \text { arbitrary } \\
& \times\left|X_{1}\right|^{\rho-1 / 2(2 s+1-r)}|W|^{\rho-1 / 2(r+1)} d X_{1} d W d Q .
\end{aligned}
$$

Then make the change of variables $Y=T Q$, where ${ }^{t} T T=X_{1}$, to obtain:

$$
\begin{aligned}
\int e^{-\sigma\left(X_{1}[Q]\right)} d Q & =\int_{Y(s, r-s)} \operatorname{real}^{-\sigma(I[Y])} d Y\left|X_{1}\right|^{-1 / 2(r-s)} \\
& =\left|X_{1}\right|^{-1 / 2(r-s)} \pi^{1 / 2 s(r-s)} \cdot \square
\end{aligned}
$$

Before concluding this section we insert a lemma on Bessel functions which proves formula (2.5) of [16] in an easy way.

Lemma 3.5. Let $A_{0}^{(n, r)}$ be such that ${ }^{t} A_{0} A_{0}=I$. Let ${ }^{t} B^{(n, r)} B$ be positive definite symmetric. And suppose that $B_{1}^{(r)} \in G L(r, \mathbf{R})$ is such that ${ }^{t} B B={ }^{t} B_{1} B_{1}$. Then

$$
\begin{aligned}
& K_{1}\left(A_{0}^{(n, r)}, B^{(n, r)}, \rho\right) \\
& \quad=\pi^{1 / 2 r(n-r)} G_{r}(\rho-1 / 2(n-r)) G_{r}(\rho)^{-1} K_{1}\left(I, B_{1}^{(r)}, \rho-1 / 2(n-r)\right) .
\end{aligned}
$$

Proof. Clearly $K\left(A_{0}, B, \rho\right)=K\left(I, B_{1}, \rho\right)$. Then use Lemma 3.3.

\section{BIBLIOGRAPHY}

1. W. L. Baily, Introductory lectures on automorphic forms, Princeton Univ. Press, Princeton, N. J., 1973.

2. K. Barner, Über die Werte der Ringklassen L-Funktionen reell-quadratischer Zahlkörper an natürlichen Argumentstellen, J. Number Theory 1 (1969), 28-64. MR 39 \#139.

3. A. Borel, Introduction to automorphic forms, Algebraic Groups and Discontinuous Subgroups, Proc. Amer. Math. Soc., vol. 9, Amer. Math. Soc., Providence, R. I., 1966. MR 34 \#7465.

4. E. Hecke, Mathematische Werke, Vandenhoeck \& Ruprecht, Göttingen, 1959, 198207. MR 21 \#3303.

5. C. S. Herz, Bessel functions of matrix argument, Ann. of Math. (2) 61 (1955), 474523. MR 16, 1107.

6. H. Jacquet, Fonctions de Whittaker associées aux groupes de Chevalley, Bull. Soc. Math. France 95 (1967), 243-309. MR 42 \#6158.

7. M. L. Karel, On certain Eisenstein series and their Fourier coefficients, Thesis, University of Chicago, 1972.

8. G. Kaufhold, Dirichletsche Reihe mit Funktionalgleichung in der Theorie der Modulfunktion 2. Grades, Math. Ann. 137 (1959), 454-476. MR 22 \#1223. 
9. M. Koecher, Über Dirichlet-Reihen mit Funktionalgleichung, J. Reine Angew. Math. 192 (1953), 1-23. MR 15, 290.

10. H. Maass, Siegel's modular forms and Dirichlet series, Lecture Notes in Math., vol. 216, Springer-Verlag, New York, 1971.

11. C. L. Siegel, Einführung in die Theorie der Modulfunktionen n-ten Grades, Math. Ann. 116 (1939), 617-657. MR 1, 203.

12. On the theory of indefinite quadratic forms, Ann. of Math. (2) 45 (1944), 577-622. MR 6, 38.

13. - Lectures on quadratic forms, Tata Institute of Fundamental Research, Bombay, 1957.

14. - Lectures on advanced analytic number theory, Tata Institute of Fundamental Research Lectures on Math., no. 23, Tata Institute of Fund. Research, Bombay, 1965. MR 41 \#6760.

15. A. A. Terras, A generalization of Epstein's Zeta function, Nagoya Math. J. 42 (1971), 173-188. MR 44 \#3970.

16. - Bessel series expansions of the Epstein zeta function and the functional equation, Trans. Amer. Math. Soc. 183 (1973), 477-486. MR 48 \#2091.

17. L. C. Tsao, Thesis, University of Chicago, 1972.

DEPARTMENT OF MATHEMATICS, UNIVERSITY OF CALIFORNIA, SAN DIEGO, LA JOLLA, CALIFORNIA 92037. 